\title{
Paroxysmal mechanical aortic valve prosthesis dysfunction: capturing the right moment
}

\author{
B. J. Sorgdrager • G. Tavilla • E. E. van der Wall • \\ A. J. H. A. Scholte
}

Published online: 27 May 2011

(C) The Author(s) 2011. This article is published with open access at Springerlink.com

A 62-year-old female presented to the emergency room under suspicion of acute myocardial infarction. While shopping she suddenly experienced severe chest pain and nearly collapsed. Her medical history revealed aortic valve replacement (Medtronic Hall $22 \mathrm{~mm}$ ) 8 years before and since 5 years repeated presentations to the emergency room because of intermittent chest pain. An initial electrocardiogram (ECG) showed sinus tachycardia with transient ST elevation in leads aVR and V1 and ST depression in leads I, II, III, aVF and V3-V6 (Fig. 1), which resolved within $5 \mathrm{~min}$. Physical examination, laboratory results and transthoracic echocardiography were unremarkable.

Under suspicion of an acute coronary syndrome, coronary angiography was performed and showed a $70 \%$ stenosis in the second diagonal branch of the left descending artery. Because of the discrepancy between the ECG at presentation and the coronary angiography,

Electronic supplementary material The online version of this article (doi:10.1007/s12471-011-0159-4) contains supplementary material, which is available to authorized users.

B. J. Sorgdrager $(\bowtie) \cdot$ E. E. van der Wall • A. J. H. A. Scholte Department of Cardiology, Leiden University Medical Center, Albinusdreef 2, P.O. Box 9600, 2300 RC, Leiden, the Netherlands e-mail: b.j.sorgdrager@lumc.nl

\section{G. Tavilla}

Department of Cardiothoracic Surgery, University Medical Center St. Radboud, Geert Grooteplein Zuid 10, P.O. Box 9101, 6500 HB, Nijmegen, the Netherlands it was decided not to perform a percutaneous coronary intervention. Immediately after the coronary angiography the patient had recurrent chest pain with remarkable ST depression. A second coronary angiography was performed which revealed non-closure of the mechanical aortic valve, causing severe aortic regurgitation (Movies 1 and 2). The patient was scheduled for valve revision. At operation dysfunction of the mechanical disk became apparent. When the disk was opened a pannus overgrowth beneath the valve was clearly visible. This was causing a block of the disk in opening position with temporarily massive aortic regurgitation (Fig. 2). After removing the mechanical prosthesis, the circular pannus overgrowth was most evident (Fig. 3). The pannus was removed and a bioprosthesis (Edwards Life Sciences Perimount Magna $23 \mathrm{~mm}$ ) was implanted. The patient made an uncomplicated recovery. She has had no recurrent chest pain 5 months after the valve replacement.

Intermittent severe aortic regurgitation due to pannus overgrowth of a mechanical prosthetic aortic valve is a serious complication and has been reported before [1-4].

The clinical presentation is varied and due to the intermittent appearance of the malfunctioning valve, the diagnosis is often difficult and can be easily overlooked. In this specific patient it was possible to diagnose this clinical problem due to the findings on angiography. However, simple cinefluoroscopy of the mechanical valve during symptoms would have diagnosed mechanical valve immobility earlier. 


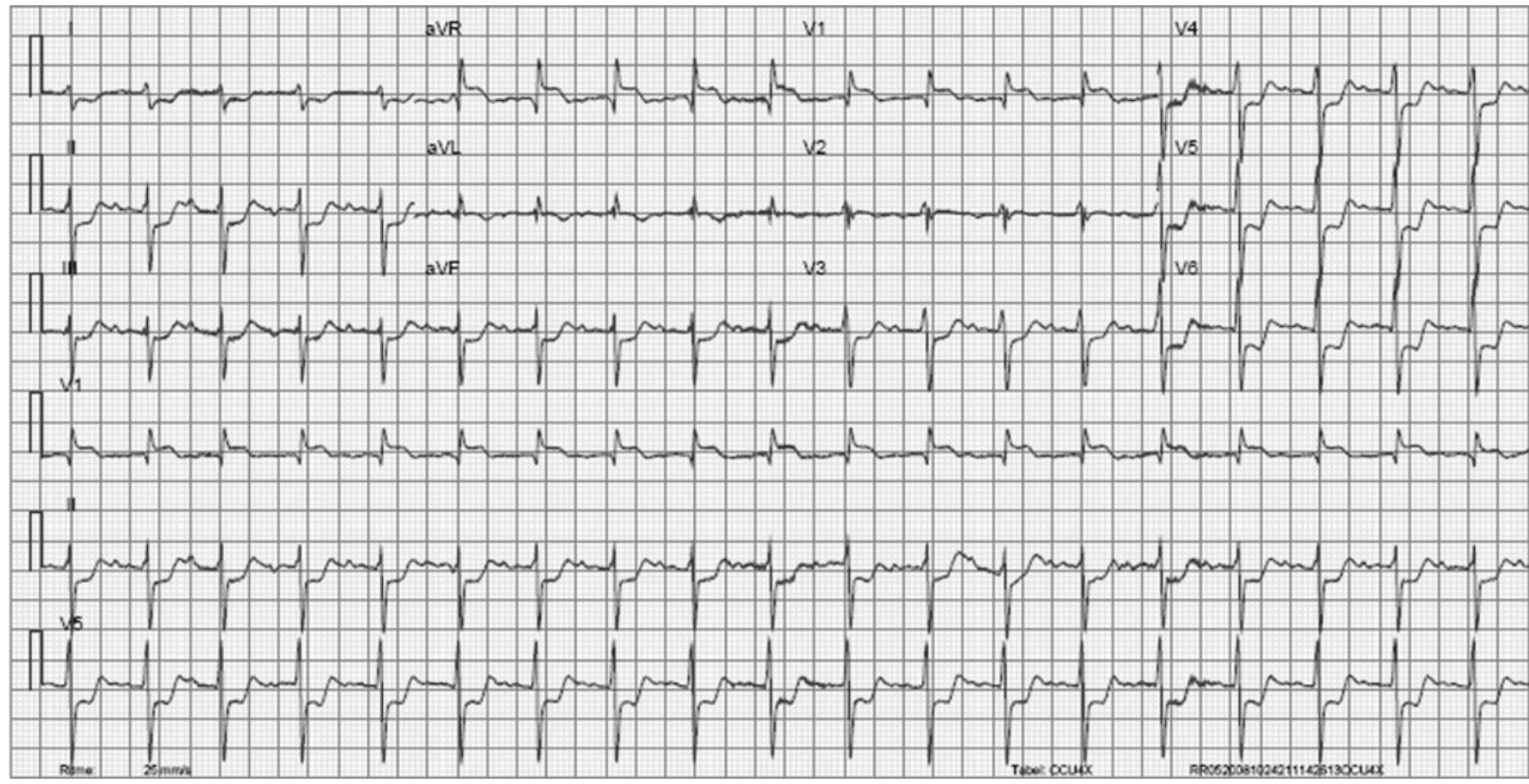

Fig. 1 ECG at presentation during chest pain, showing sinus tachycardia with ST elevation in leads aVR and V1 and ST depression in leads I, II, III, aVF and V3-V6

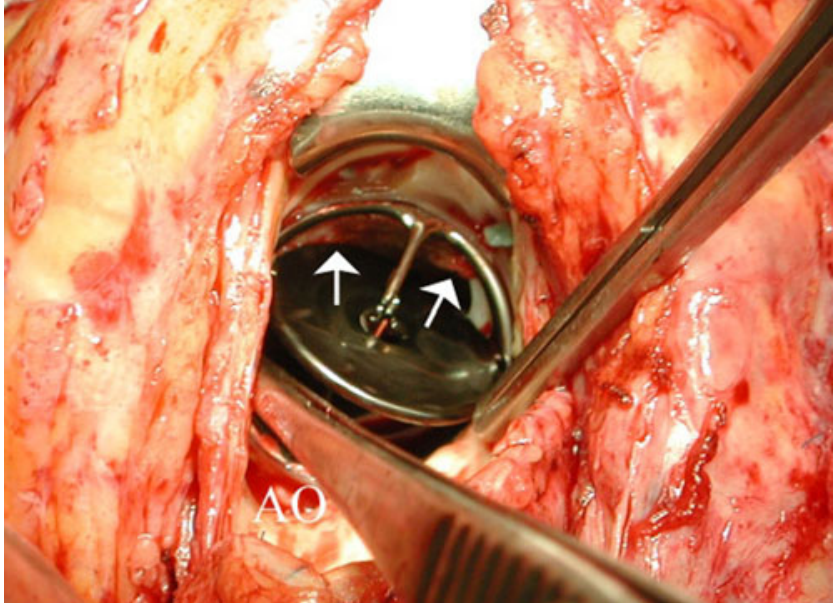

Fig. 2 The mechanical prosthesis is opened. A pannus overgrowth is visible behind the prosthesis (indicated by arrows). AO: ascending aorta

Open Access This article is distributed under the terms of the Creative Commons Attribution Noncommercial License which permits any noncommercial use, distribution, and reproduction in any medium, provided the original author(s) and source are credited.

\section{References}

1. Fan D, Makaryus AN, Hamik A, et al. Prosthetic valve dysfunction presenting as intermittent acute aortic regurgitation. Echocardiography. 2008;25(8):925-7.

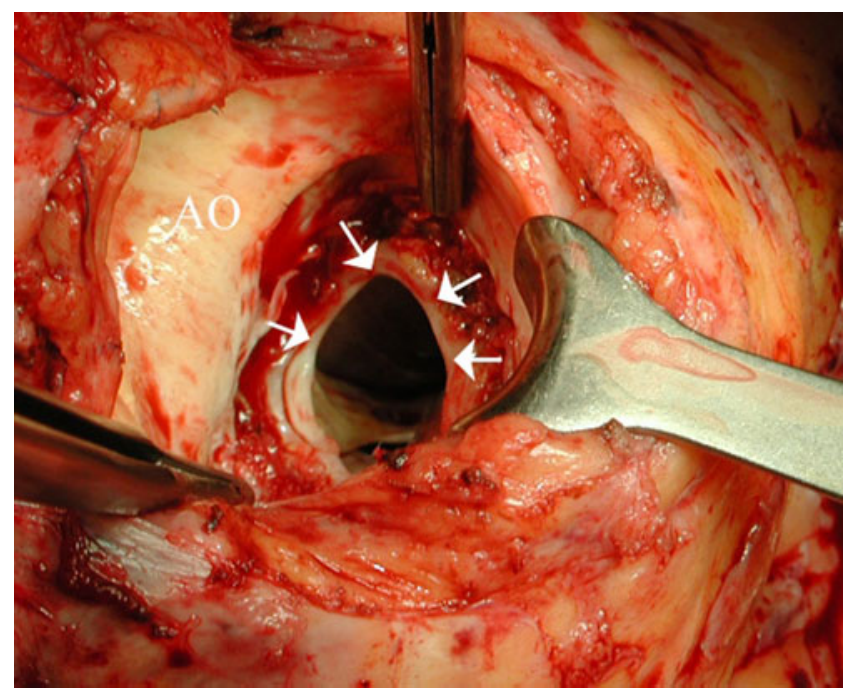

Fig. 3 After removing the mechanical prosthesis, the circular pannus is entirely visible (indicated by arrows)

2. Karagiannis SE, Karatasakis G, Spargias K, et al. Intermittent acute aortic valve regurgitation: a case report of a prosthetic valve dysfunction. Eur J Echocardiogr. 2008;9(2):291-3.

3. Cianciulli TF, Saccheri MC, Lax JA, et al. Intermittent acute aortic regurgitation of a mechanical bileaflet aortic valve prosthesis: diagnosis and clinical implications. Eur J Echocardiogr. 2009;10 (3):446-9.

4. Tanaka H, Horinouchi N, Hizukuri K, et al. Stuck prosthetic aortic valve resulting in intermittent chest pain and ST depression: a case report. J Cardiol. 2000;36(4):263-7. 